\title{
Modern surgical treatment of malignant skin melanoma: A brief literature
}

\section{overview}

\author{
Strahil Strashilov ${ }^{1}$, Angel Yordanov²
}

1. Department of Plastic Restorative, Reconstructive and Aesthetic Surgery, Medical University Pleven, Bulgaria

2. Department of Gynecologic Oncology, Medical University Pleven, Bulgaria

\section{Corresponding Author:}

Angel Danchev Yordanov

Clinic of Gynecologic Oncology, Medical University Pleven, Georgi Kochev 8A, Bulgaria

Email: angel.jordanov@gmail.com

\section{ABSTRACT}

\section{Background}

Malignant melanoma (MM) of the skin is a rare, highly malignant tumour, affecting younger age. Its incidence rate has been rising as compared to all malignant neoplasms -5 per cent of all newly diagnosed cancers in men, and 6 per cent of those in women.

\section{Aims}

The aim of the literature review is to present the contemporary tendencies in the surgical treatment and monitoring of patients with malignant melanoma of the skin.

\section{Methods}

Systematic Literature Review Made By Google and Science Direct.com Search Engines. Publications and guidelines in English, including the newest aspects in the overall care of patients with malignant melanoma of the skin. Information for the indications and contraindications of performing a sentinel biopsy.

\section{Results}

The study established that the most modern surgical treatment of a malignant melanoma of the skin includes: primary tumour biopsy, sentinel biopsy of the regional lymph nodes with wide re-excision of the affected area, usually with a radius of $2 \mathrm{~cm}$, and the removal of local recurrences, lymph and distant organ metastases. When it comes to monitoring, it has to be done according to the contemporary worldwide guidelines.

\section{Conclusion}

Successful treatment of skin MM is in direct correlation to keeping up with the most modern tendencies.

\section{Key Words}

Malignant melanoma, surgical treatment, sentinel lymph node biopsy

\section{What this review adds:}

\section{What is known about this subject?}

Malignant melanoma ( $\mathrm{MM}$ ) of the skin is a rare, highly malignant tumour, affecting younger age. Its incidence rate has been rising as compared to all malignant neoplasms.

\section{What new information is offered in this review?}

This review explores modern management of malignant melanoma.

3. What are the implications for research, policy, or practice?

Improvements in the management of malignant melanoma.

\section{Introduction}

Malignant skin melanoma (MM) is a rare and highly malignant tumour, affecting younger age. Its incidence rate is growing faster than that of other malignant neoplasms. If compared to other newly diagnosed malignancies, it accounts for 5 per cent of these in men and 6 per cent in women. ${ }^{1}$ 
Modern treatment for $\mathrm{MM}$ includes tumour and sentinel regional lymph node biopsy, radical excision of the tumour bed eventually followed by lymph dissection, and surgical removal of distant metastases.

Sentinel regional lymph node biopsy is an essential part of treatment and is crucial for a prognosis.

\section{Body of the minireview}

Biopsy of the MM and a histopathological examination

- Incisional biopsy implies excising only a part of a larger tumour mass

- Excisional biopsy - the whole melanoma is removed, together with 1 to $3 \mathrm{~mm}$ of visually healthy surrounding skin tissue. ${ }^{2}$ No radical tumour excision is performed in order to avoid disconnecting lymph vessels. Such an excision would vitiate a following sentinel biopsy of the regional lymph nodes.

- Pathological examination of the MM implies defining the thickness of the melanoma is defined after Breslow and the depth of tumour invasion after Clark. ${ }^{3}$

\section{Radical re-excision of the tumour bed}

This has to be scheduled within four to six weeks after diagnosing the MM. ${ }^{4}$ The skin around the primary tumour biopsy site is removed after the sentinel biopsy. The distance between the border of re-excision and the scar line from the previous biopsy depends on the thickness of the MM as established by the histological investigation. 5,6

\section{Sentinel biopsy of regional lymph nodes}

This implies removal of the first lymph node/s with metastases from a malignant neoplasm. The histological examination provides accurate prognosis regarding the degree of involvement of the remainder of the lymph nodes. ${ }^{7}$ In 1992, Donald Morton et al. introduced sentinel biopsy in skin MM to avoid post-operative complications of the skin, aiming to avoid the common post-operative complications and prevent the lack of effect in case of a negative sentinel lymph node associated with the routine total lymph dissection applied at that time. ${ }^{8}$

- Indications for performing sentinel biopsy presence of clinically negative regional lymph nodes

$$
\begin{aligned}
& \text { - } \quad \text { tumour thickness over } 1 \mathrm{~mm} \\
& \text { - in cases of tumours thickness between }
\end{aligned}
$$

0.76 and $1 \mathrm{~mm}$, sentinel biopsy is recommendable only if additional factors are present, such as tumour ulcerations, high mitotic index, young patients, etc. ${ }^{9,10}$

- Indications against sentinel biopsy are:

- $\quad$ the prevalent number of the MMs are less than $0.75 \mathrm{~mm}$ thick;

- clinically and puncture-biopsy positive regional lymph nodes;

- history of lymph biopsy;

- extended biopsy performed at the primary

focus of the malignancy;

$$
\begin{aligned}
& \text { - lack of a team specialized in sentinel } \\
& \text { biopsy. }
\end{aligned}
$$

- $\quad$ Sentinel lymph biopsy technique

Sentinel lymph node biopsy procedures include, as follows: injecting 0.28 to $10 \mu \mathrm{Ci}$ of a radiopharmaceutical agent (99Tc sulphur colloid) at 4 intradermal spots around the scar of a previous biopsy of the $\mathrm{MM}$; examining the patients in a gamma camera to make a lymphoscintigraphic map, visualizing the regional lymph drain, location and number of sentinel lymph nodes and presence or absence of intransitive lymph nodes; ${ }^{12,13}$ in the operating room, $1 \mathrm{ml}$ of lymphotropic dye (Patent Blue V) is applied intradermally at ten locations around the scar. After 6 to 10 minutes, the areas marked on the lymphoscintigraphic map are explored to find the sentinel lymph node. The node is blue and its location corresponds to the spot as indicated on the map. After disconnecting the supplying vessels, the node is removed and sent for histological analysis to search for metastases. $^{14}$

One other method of sentinel biopsy employs intraoperative use of a manual gamma camera to spot a lymph node after a lymphoscintigraphic map of the area has been made. There is also an option to use the gamma camera pre- and intraoperatively, without a preliminary lymphoscintigraphic map. ${ }^{15}$

Recent data from clinical studies on sentinel biopsy (MSLT 1 and 2) have shown that it is crucial for the complex treatment of the MM. Four major points are in support of its role:

1. The result obtained using sentinel lymph node is recognized as one of the major prognostic factors.

2. Lymph dissection following positive sentinel biopsy in patients with certain thin, all intermediate, and thick melanomas significantly increases the survival rates in good health.

3. The result from biopsy is a cornerstone of an effective adjuvant therapy.

4. Biopsy of a sentinel lymph node is a very sparing surgical intervention. ${ }^{16,17}$ 


\section{Lymph dissection of regional lymph nodes}

Removal of the whole regional lymph pool is undertaken when it affected by the MM metastatic process, which has been proved histologically, by a cytological examination of a sentinel lymph node or another lymph node found positive after clinical or [an apparatus examination] a gamma camera examination. ${ }^{18}$ According to data from currently reported studies (MSLT 1 and 2), lymph dissection immediately after finding a metastatic sentinel lymph node increases the survivability of patients in good health compared to those with dissection, performed after clinically positive for metastases lymph nodes. ${ }^{16,17}$

Histopathological examination of removed sentinel and other lymph nodes.

Once removed, both sentinel and other lymph nodes are examined with serial section immunohistochemistry, using MM-specific markers (S100, Melan A; HMB-45) for metastases, micrometastases or isolated tumour cells. ${ }^{19}$ As the depth of MM by Breslow increases, the percentage of metastasis-positive sentinel lymph nodes also increases. ${ }^{20}$

\section{Additional surgical treatment}

It is only performed in the presence of distant metastases, local recurrences, etc., and it includes surgical interventions of various extents.

\section{Disease staging}

Four clinical stages are determined, as specified by the TNM classification. Precise staging is a crucial for the success of treatment undertaken.

\section{Adjuvant treatment}

This includes immunotherapy, target therapy, vaccine therapy, chemotherapy, radiotherapy, etc.

\section{Follow-up observation}

Follow-up is also a crucial part of complex treatment. It includes all clinical, laboratory and instrumental examinations that a patient with a proven diagnosis of MM has undergone. In case of abnormal findings, additional surgical and/or non-surgical treatment may be assigned.

\section{Conclusion}

Successful treatment of skin MM is in direct correlation to keeping up with the most modern tendencies.

Contemporary surgical treatment is a cornerstone in complete treatment of MM. One of the most reliable prognostic factors is the result obtained by sentinel lymph node biopsy.
Performing lymph dissection after positive sentinel biopsy in patients with some thin, all intermediate and thick melanomas, significantly increases the survival rate in good health. The result of the sentinel biopsy is the cornerstone of an effective adjuvant therapy. Sentinel biopsy is a very sparing surgical intervention.

\section{References}

1. Balabanova M, Saleva M. Melanoma Malignum. Medinfo. 2016;(04):24-29.

2. Coit D, Andtbacka R, Bichakjian C, et al. Melanoma. J Natl Compr Canc Netw. 2009;7:250-75.

3. Balch C, Soong S, Gershenwald J, et al. Prognostic factors analysis of 17,600 melanoma patients: Validation of the American Joint Committeeon Cancer melanoma staging system. J Clin Oncol. 2001;19(16):3622-34.

4. Garbe C, Peris K, Hauschild A, et al. On behalf of the European Dermatology Forum (EDF) the European Association of Dermato-Oncology (EADO) and the European Organisation for Research and Treatment of Cancer (EORTC). Diagnosis and treatment of melanoma. European consensus-based interdisciplinary guideline e Update 2016. Eur J Cancer. 2016;63:201-217.

5. Lens $M$, Nathan $P$, Bataille $V$. Excision margins for primary cutaneous melanoma: updated pooled analysis of randomized controlled trials. Arch Surg. 2007;142:885-93.

6. Veronesi U, Cascinelli N. Narrow excision (1-cm margin): a safe procedure for thin cutaneous melanoma. Arch Surg. 1991;126:438-41.

7. Balch C, Gershenwald J, Soong S, et al. Final version of 2009 AJCC melanoma staging and classification. J Clin Oncol. 2009;27(36):6199-206.

8. Baytchev G. Biopsy of sentinel lymph nodes in malignant melanoma. My Surgery. 2011.

9. Gershenwald J, Mansfield P, Lee J, et al. Role for lymphatic mapping and sentinel lymph node biopsy in patients with thick (< or $=4 \mathrm{~mm}$ ) primary melanoma. Ann Surg Oncol. 2000;7:160-5.

10. Ferrone C, Panageas K, Busam K, et al. Multi variate prognostic model for patients with thick cutaneous melanoma: importance of sentinel lymph node status. Ann Surg Oncol. 2002;9:637-45.

11. National Comprehensive Cancer Network. Melanoma: Clinical Practice Guidelines in Oncology. Version 2. 2007. National Comprehensive Cancer Network.

12. Morton D, Thompson J, Essner R, et al. Validation of the accuracy of intraoperative lymphatic mapping and sentinel lymphadenectomy for early stage melanoma: a multicenter trial; multicenter selective lymphadenectomy trial group. Ann Surg. 1999;230:453- 
65.

13. Mattsson J, Bergkvist L, Abdiu A, et al. Sentinel node biopsy in malignant melanoma: Swedish experiences 1997- 2005. Acta Oncol. 2008;47:1519-25.

14. Essner R. Surgical treatment of malignant melanoma. Surg Clin N Am. 2003;83:109-156.

15. Sapienza M, Tavares M, Endo I, et al. The role of sentinel node mapping in malignant melanoma: experience with 99mTc-phytate and a review of the literature.

16. Spillane A, Read R, Thompson J. Sentinel node biopsy should be the standard of care for patients with intermediate and thick melanomas. Aust Fam Physician. 2015;44(8):604-6.

17. Morton D, On behalf of the MSLT Study Group. Overview and update of the phase III Multicenter Selective Lymphadenectomy Trials (MSLT-I and MSLT-II) in melanoma. Clin Exp Metastasis. 2012;29(7):699-706.

18. Morton D, Wanek L, Nizze J, et al. Improved long- term survival after lymphadenectomy of melanom metastatic to regional nodes. Analysis of prognostic factors in 1134 patients from the John Wayne Cancer Clinic. Ann Surg. 1991;214(4):491-9.

19. Byron E, Randall P, Xing $Y$, et al. The importance of sentinel lymph node biopsy in patients with thin melanoma. Arch Surg. 2008;143(9):892-900.

20. Lens $M$, Dawes $M$, Newton-Bishop J, et al. Tumour thickness as a predictor of occult lymph node metastases in patients with stage I and II melanoma undergoing sentinel lymph node biopsy. $\mathrm{Br} J$ Surg. 2002;89(10):1223-1227.

\section{PEER REVIEW}

Not commissioned. Externally peer reviewed.

\section{CONFLICTS OF INTEREST}

The authors declare that they have no competing interests.

\section{FUNDING}

This publication is supported by Project N BG05M2OP0012.009-0031-C01

\section{ETHICS COMMITTEE APPROVAL}

Local ethics committee, Medical university Pleven city; Bulgaria 